\title{
HIV persists throughout deep tissues with repopulation from multiple anatomical sources
}

\author{
Antoine Chaillon, ${ }^{1}$ Sara Gianella, ${ }^{1}$ Simon Dellicour, ${ }^{2,3}$ Stephen A. Rawlings, ${ }^{1}$ Timothy E. Schlub, ${ }^{4}$ Michelli Faria De Oliveira, ${ }^{1}$ \\ Caroline Ignacio, ${ }^{1}$ Magali Porrachia, ${ }^{1}$ Bram Vrancken, ${ }^{3}$ and Davey M. Smith ${ }^{1}$ \\ 'Department of Medicine, UCSD, La Jolla, California, USA. ${ }^{2}$ Spatial Epidemiology Lab (SpELL), Université Libre de Bruxelles, Bruxelles, Belgium. ${ }^{3}$ KU Leuven, Department of Microbiology and Immunology, \\ Rega Institute, Laboratory of Computational and Evolutionary Virology, Leuven, Belgium. ${ }^{4}$ University of Sydney, Faculty of Medicine and Health, Sydney School of Public Health, Sydney, Australia.
}

BACKGROUND. Understanding HIV dynamics across the human body is important for cure efforts. This goal has been hampered by technical difficulties and the challenge of obtaining fresh tissues.

\begin{abstract}
METHODS. This observational study evaluated 6 individuals with HIV ( $n=4$ with viral suppression using antiretroviral [ART] therapy; $\boldsymbol{n}=\mathbf{2}$ with rebound viremia after stopping ART), who provided serial blood samples before death and their bodies for rapid autopsy. HIV reservoirs were characterized by digital droplet PCR, single-genome amplification, and sequencing of fulllength (FL) envelope HIV. Phylogeographic methods were used to reconstruct HIV spread, and generalized linear models were tested for viral factors associated with dispersal.
\end{abstract}

RESULTS. Across participants, HIV DNA levels varied from approximately 0 to 659 copies/10 ${ }^{6}$ cells (IQR: 22.9-126.5). A total of 605 intact FL env sequences were recovered in antemortem blood cells and across 28 tissues (IQR: 5-9). Sequence analysis showed (a) the emergence of large, identical, intact HIV RNA populations in blood after cessation of therapy, which repopulated tissues throughout the body; (b) that multiple sites acted as hubs for HIV dissemination but that blood and lymphoid tissues were the main source; (c) that viral exchanges occurred within brain areas and across the blood-brain barrier; and (d) that migration was associated with low HIV divergence between sites and greater diversity at the recipient site.

CONCLUSION. HIV reservoirs persisted in all deep tissues, and blood was the main source of dispersal. This may explain why eliminating HIV susceptibility in circulating T cells via bone marrow transplants allowed some individuals with HIV to experience therapy-free remission, even though deeper tissue reservoirs were not targeted.

TRIAL REGISTRATION. Not applicable.

FUNDING. NIH grants P01 AI31385, P30 AI036214, Al131971-01, Al120009AI036214, HD094646, AI027763, Al134295, and Al68636.

\section{Introduction}

Upon transmission to a new host, HIV-1 disseminates to the lymph nodes within days and to the bloodstream and throughout the human body within 1 to 2 weeks (1-4). In this process, HIV establishes reservoirs throughout the body including in the CNS, lymphoid tissues (spleen, thymus, lymph nodes, gut-associated lymphoid tissue), bone marrow, lungs, kidneys, liver, adipose tissue, gastrointestinal tract, and genitourinary systems (5). The persistence of replication-competent HIV in these ana-

\section{Related Commentary: p. 1611}

Authorship note: $A C$ and SC contributed equally to this work. BV and DMS contributed equally to this work.

Conflict of interest: The authors have declared that no conflict of interest exists. Copyright: (c) 2020, American Society for Clinical Investigation. Submitted: November 6, 2019; Accepted: December 19, 2019;

Published: February 24, 2020

Reference information: / Clin Invest. 2020;130(4):1699-1712.

https://doi.org/10.1172/JCl134815. tomical sites, even in the setting of longstanding, potent antiretroviral therapy (ART) that suppresses HIV replication, is the main barrier to curing $\operatorname{HIV}(6)$.

Despite extensive studies in humans $(5,7-18)$, much remains unclear about HIV reservoirs that persist during ART (19-23). In part, this is because of technical limitations and limited access to appropriately collected tissues for such studies. In general, most studies aiming to characterize HIV DNA reservoirs in persons with HIV (PWH) have necessarily focused on blood, with a few other compartments that are relatively easy to sample such as the gut, through endoscopy $(5,10,11,24)$, the genital tract, through genital secretions $(12,25-30)$, or the CNS, through cerebrospinal fluid (CSF) (31-37). Of note, one study in PWH diagnosed with cancer allowed the analysis of HIV reservoirs in selected anatomic tissues collected during autopsy $(38,39)$. More recently, De Scheerder et al. investigated the origins of HIV rebound, and although this study was limited by the number of anatomical sites, they showed that viral rebound originated from diverse cellular and tissue reservoirs (40). To address tissue availability, a perimortem observational 


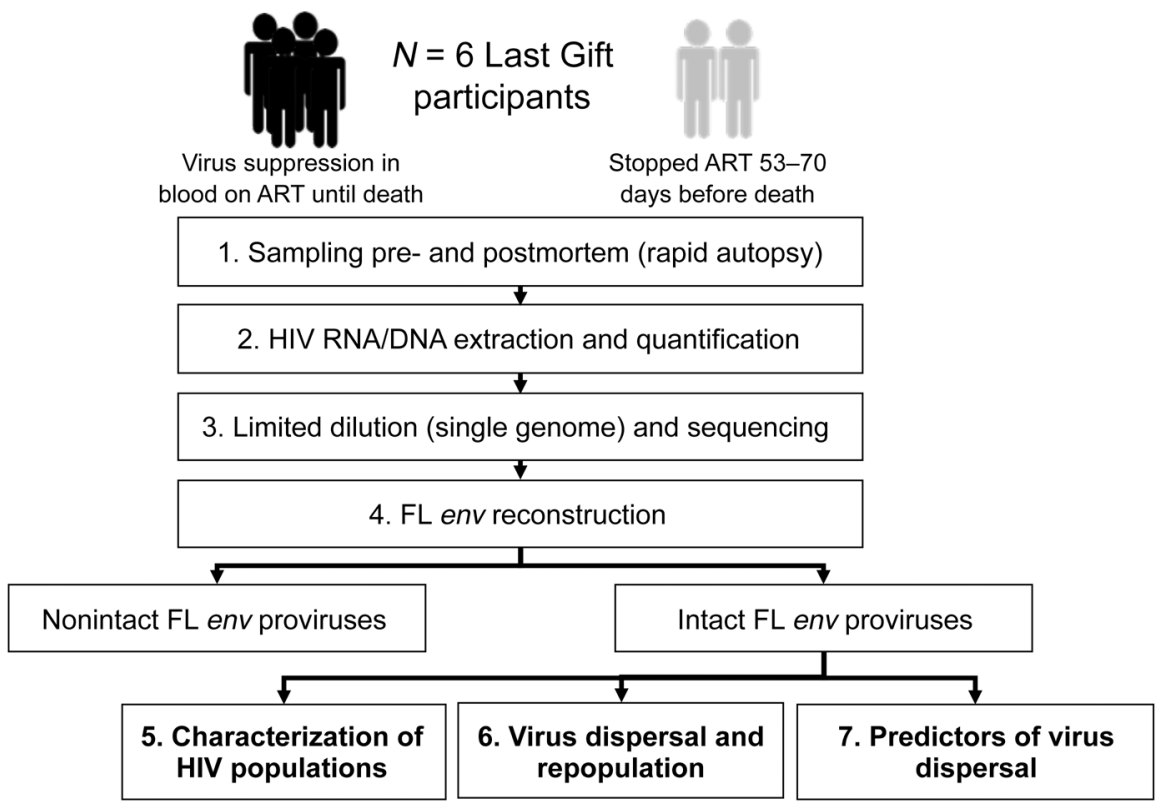

Figure 1. Flow diagram. Six participants enrolled in the Last Gift cohort ( $n=2$ participants who stopped ART; $n=4$ participants who remained virally suppressed on ART until death) were included in this study. (1) Premortem blood plasma ( $n=2$ participants) and PBMC samples ( $n=6$ participants) were collected before death, and tissues were collected during the rapid autopsy procedure. (2) HIV RNA and DNA were extracted from blood plasma and PBMCs and tissues for quantification of HIV DNA/RNA (ddPCR). (3 and 4) HIV FL envelopes were sequenced via single-genome amplification and sequencing. Intact $F L$ env sequences from all samples were used to (5) characterize the HIV populations within each compartment and in blood, (6) assess viral dispersal across tissues using Bayesian phylodynamic models, and (7) evaluate factors associated with viral dispersal. research cohort, called the Last Gift, was developed and enrolled PWH diagnosed with a terminal illness from a non-HIV condition. Participants consented to blood sampling before death and donated their bodies for rapid autopsy after death (41-43). This allowed us to collect antemortem blood and postmortem tissues across 28 anatomical compartments from 6 participants. Postmortem tissues were collected within 6 hours of death for maximal preservation of tissue and viral integrity (43). In the Last Gift cohort, some participants decided that they no longer wanted to continue their ART in the days and weeks before death. This provided the opportunity to observe and characterize rebounding viral populations in blood and compare them with viral populations found in tissues.

To characterize the HIV reservoirs in collected specimens, we combined new technologies that could deep sequence near-fulllength (near-FL) env HIV genomes and sensitively quantify HIV DNA $(44,45)$ using established molecular epidemiology inference methods to assess the viral diversity, divergence, predicted cellular tropism, replication competence, compartmentalization, and migration across the human body.

\section{Results}

Cohort. The design of the study is summarized in Figure 1. This study evaluated 6 male participants (see Supplemental Table 1 and Supplemental Figure 1; supplemental material available online with this article; https://doi.org/10.1172/JCI134815DS1) whose median age at the time of death was 57.5 years (range: $52-72$ years). All participants initiated ART during chronic infection and were virally suppressed for a mean of 7.5 years before enrolling in the Last Gift study (range: 3-14 years). Two participants, LG01 and LG04, voluntarily interrupted ART 53 days and approximately 70 days before death, respectively. The last HIV RNA blood plasma levels before death for LG01 and LG04 were 280 copies/mL and 48,000 copies/mL, respectively. Participants LG03, LG05, LG06, and LG08 had undetectable HIV RNA in blood plasma $(<20$ copies $/ \mathrm{mL})$ until death and at the time of the autopsy. The median $\mathrm{CD} 4^{+} \mathrm{T}$ cell count before death was $338 / \mathrm{mm}^{3}$ (range: $174-868 / \mathrm{mm}^{3}$ ).
HIV DNA levels. Overall, HIV DNA levels varied in sampled blood and tissues from approximately 0 -658.8 copies $/ 10^{6}$ cells (median = 55.9, IQR: 22.9-126.5) (Supplemental Table 2, Figure 2F, and Figure 3). As expected, HIV DNA levels were lowest in CNS samples varying from a range of $0-4.6$ copies $/ 10^{6}$ cells in the occipital lobe to $1.8-33.6$ copies $/ 10^{6}$ cells in the frontal lobe. Although the sample size was small, we detected no discernible difference in HIV DNA levels in tissues between the participants who interrupted ART and those who did not $(P>0.05)$.

Defective proviruses (HIV DNA). Across all samples from all participants, we recovered 676 single-genome FL env sequences (Supplemental Table 2). Hypermutated and otherwise defective (i.e., nonintact) proviruses (FL env) accounted for $10.5 \%$ of all sequences ( 71 of 676 ), varying from $0 \%-18.3 \%$ of all FL env sequences in a given tissue (Supplemental Table 2 and Figure 2B). Nonintact FL env sequences were excluded from further analyses, resulting in 605 evaluated intact FL env sequences (55-152 sequences/participant, with a median of 7 sequences/ site; IQR: 5-9). Overall, we found evidence that the proportion of genetically intact FL env variants varied between anatomical compartments $(P=0.016)$; however, the pattern of this variation was not consistent across participants $(P=0.96)$. To further investigate this, the 28 compartments were collapsed to 11 semirelated categories (blood plasma, cardiac serum, gut, CNS, kidney, liver, lymphoid, pancreas, PBMC, pericardial adipose, and genital tract), and we still did not detect a pattern. We also found weak evidence for a difference in the proportion of intact FL env variants across participants $(P=0.04)$, but this association was weakened further after adjusting for anatomical compartment $(P=0.27)$ and anatomical compartment category $(P=$ 0.051). The prevalence of intact FL $e n v$ variants for each participant and anatomical compartment is presented in Figure 2 and Supplemental Table 2.

Viral diversity and clonality. Viral diversity of HIV RNA populations in blood and HIV DNA populations in tissues varied between tissues across participants and across tissues within 
A

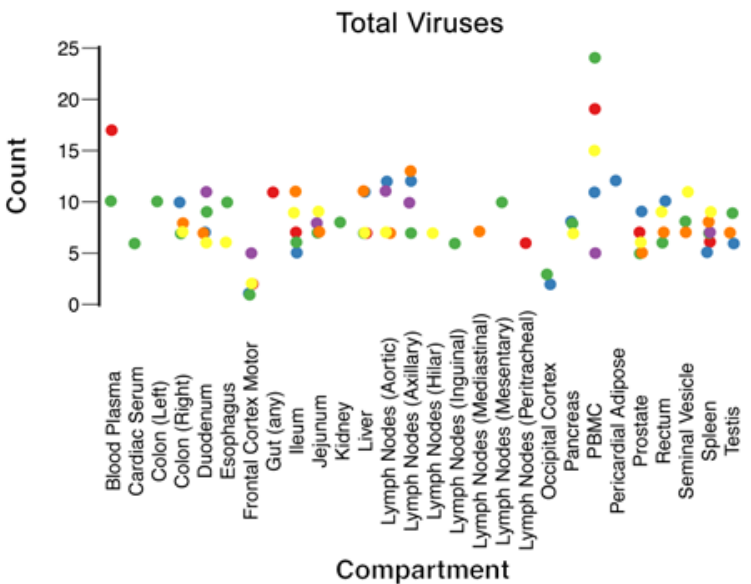

C
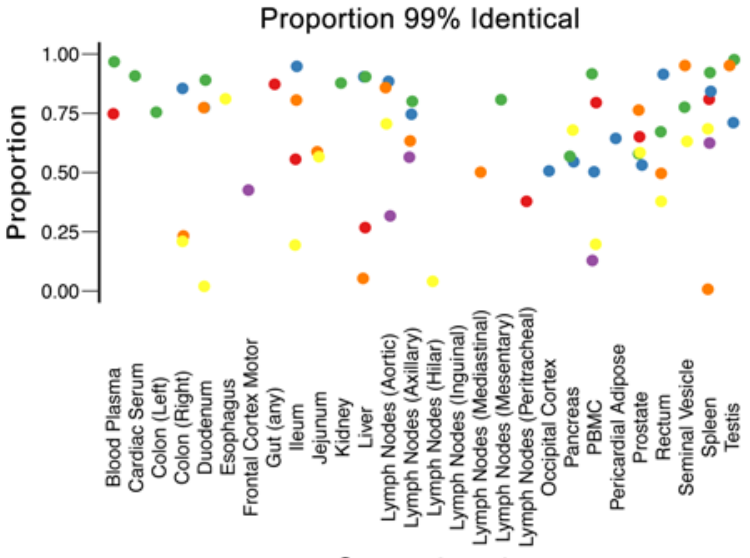

Compartment

\section{E}
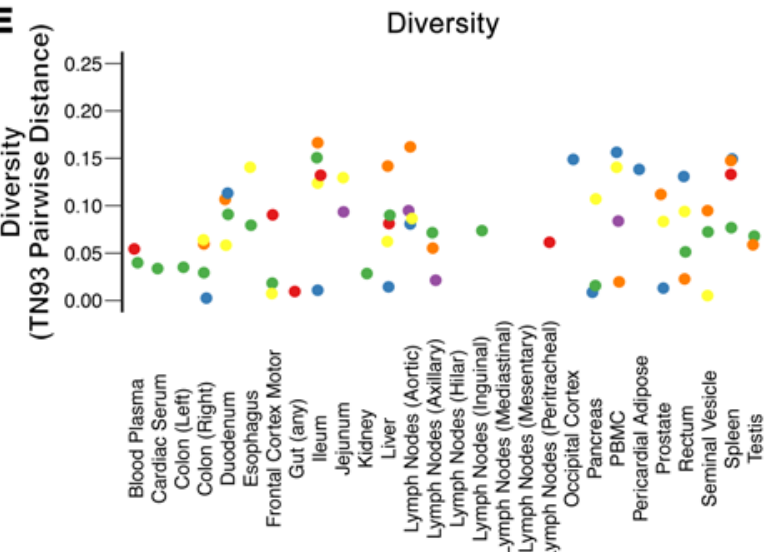

Compartment
PIDs: : LG01 : LG03 - LG05 LG06
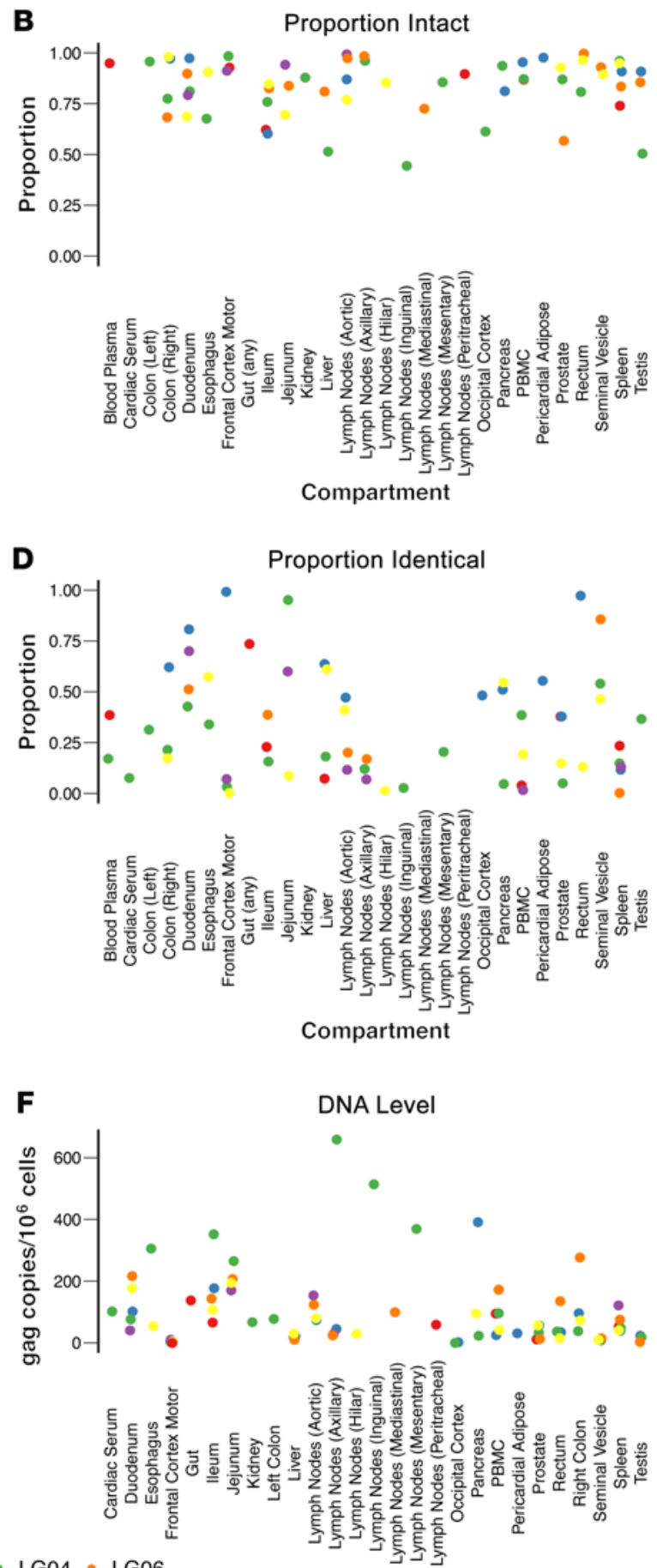

Compartment

Figure 2. Characteristics of the HIV populations within each compartment. Scatter dot plots sorted by sampled compartment and colored by participants with the $y$ axis showing (A) the total number of sequences; (B) the proportion of intact sequences; (C) the proportion of nearly identical sequences (any sequences with $\geq 99 \%$ nucleotide identity with at least 1 other sequence from the same participant); (D) the proportion of identical sequences (100\%); (E) the pairwise genetic distance between sequences; and (F) the levels of HIV DNA. The pairwise genetic distance between sequences from a compartment was measured using the TN93 algorithm (46). HIV DNA levels were quantified by ddPCR. Copy numbers were calculated as the mean of 3 replicate PCR measurements and normalized to 1 million cells, as determined by RPP30 assay (total cell count) $(94,95)$. See Methods for details. PIDs, participant identifiers.

participants (Figure 2E, Supplemental Table 2, and Supplemental Figure 2). In participants who decided to stop therapy, most of the rebounding intact HIV RNA in blood (65\% and $80 \%$ of variants) was more than $99 \%$ identical, with the mean pairwise nucleotide
Tamura-Nei 93 (TN93) distance (46) of 0.015 for LG01 and 0.001 for LG04. Overall, viral diversity differed significantly across participants $(P=0.057)$ but not across tissues $(P=0.51)$; however, our power to detect differences across tissues was poor due to the 

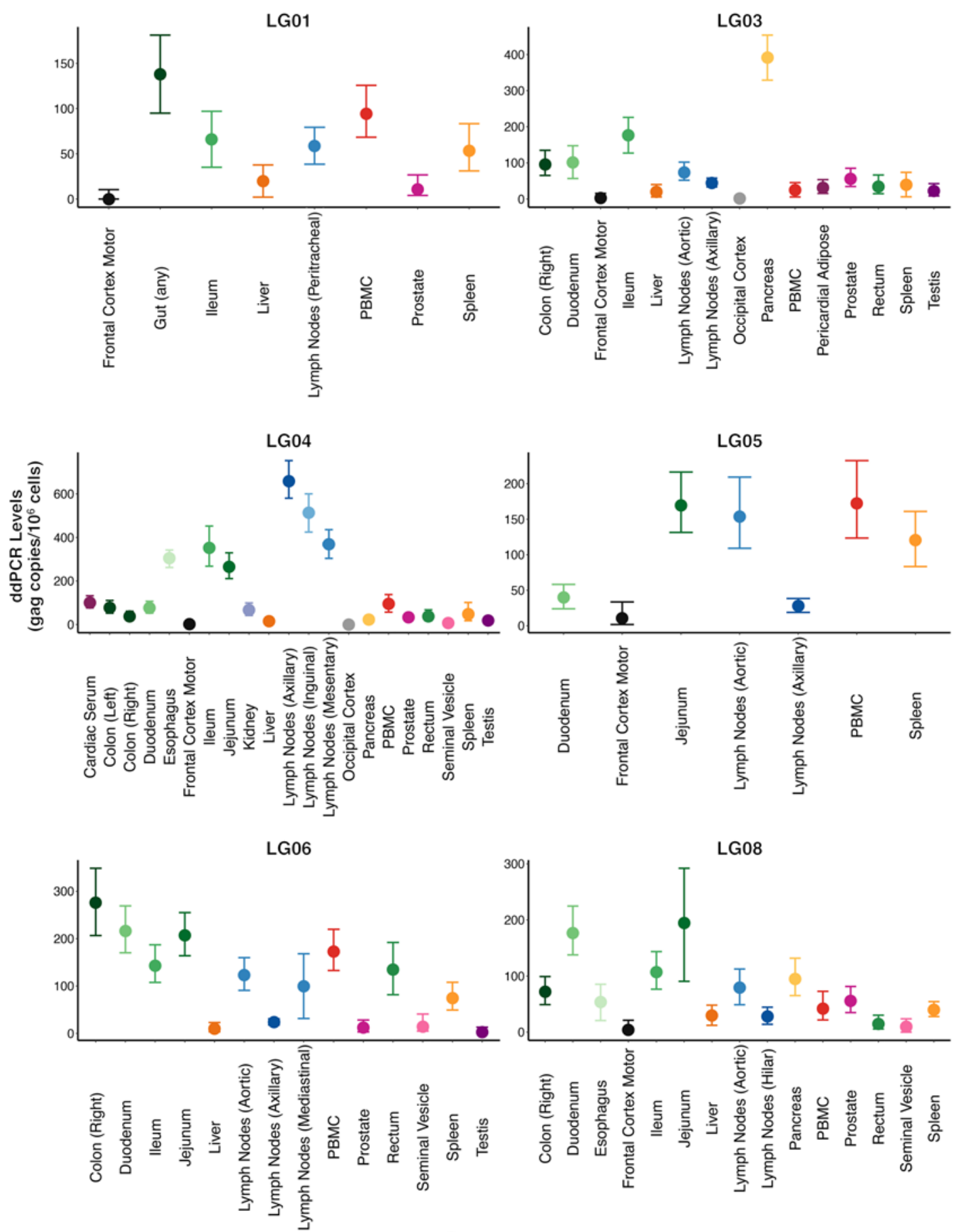

Compartments

Figure 3. HIV DNA levels in compartments for each participant. HIV DNA levels were quantified by ddPCR. Copy numbers were calculated as the mean of 3 replicate PCR measurements and normalized to 1 million cells, as determined by RPP30 assay (total cell count) (94, 95). See Methods for details. The dots and horizontal bars represent the mean and range (minimum and maximum) of the HIV DNA levels.

large number of compartments and small number of participants. Considering this, we also looked at individual tissue comparisons with blood plasma HIV RNA populations and found that viral diversity was overall higher for all HIV DNA in tissues, but the higher viral diversity was only significant for prostate $(P=0.046)$, rectum $(P=0.045)$, right colon $(P=0.013)$, spleen $(P=0.013)$, and occipital cortex $(P=0.006)$.
We also found evidence of identical intact FL env proviruses within and across tissues. For example, monoclonal FL env populations (100\% identical) were found in ileum (3 of 3 intact FL env sequences sampled) in LG03; jejunum (7 of 7) in LG04; and testes (6 of 6) in LG06. We also found identical FL env sequences across compartments, with LGO3 having 30 identical intact envelopes recovered from 8 anatomical compartments and LGO4 having 14 


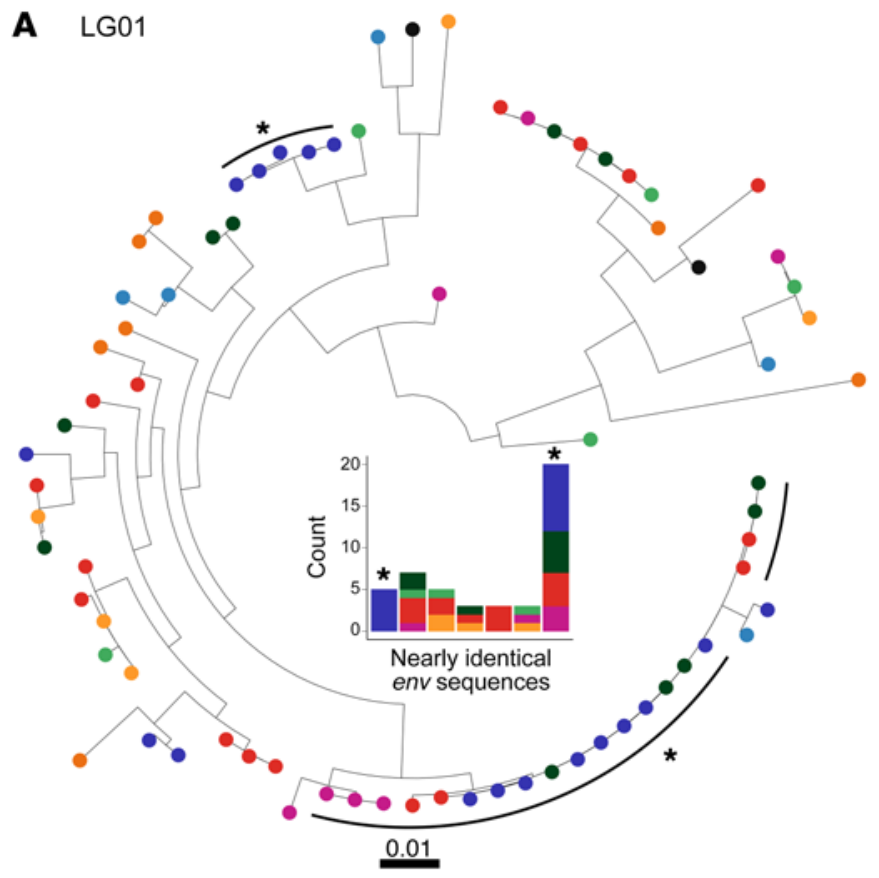

B LG03

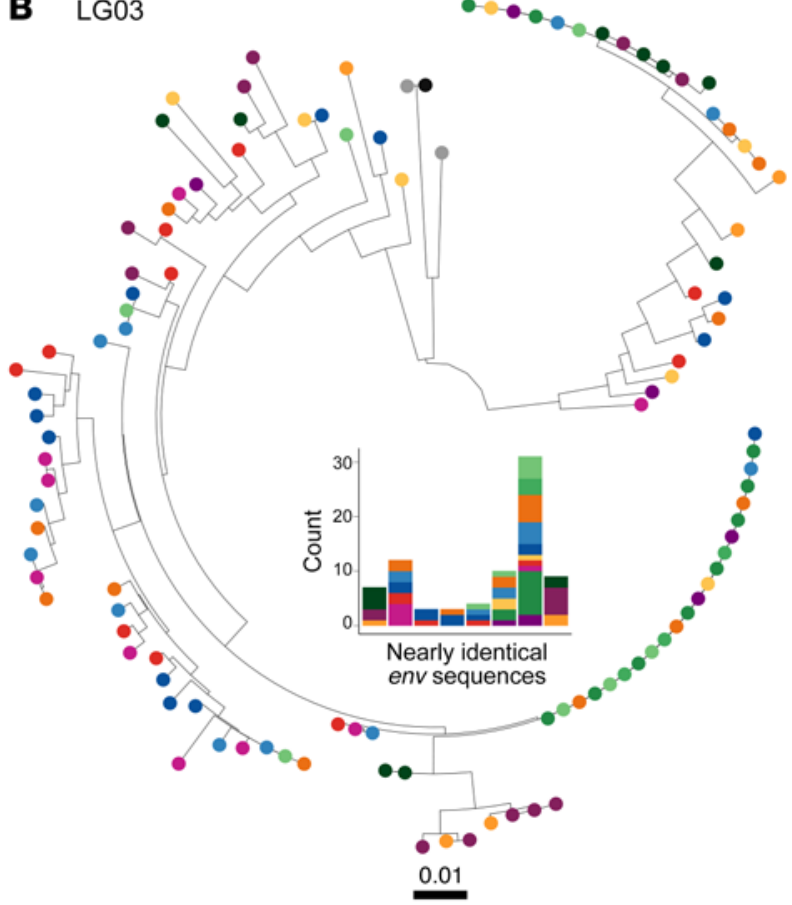

identical intact envelopes from 5 tissue compartments. We also found a large, nearly identical population ( $\geq 99 \%$ identical) of 25 FL env proviruses sampled across 10 compartments in participant LG08 (Figure 4 and Supplemental Figure 4). The proportion of identical FL env sequences varied significantly across participants $(P<0.01)$, with the following proportions of identical FL env variants throughout the body: LG01 (21 of 75, 28\%); LG03 (54 of 113, 48\%); LGO4 (42 of 152, 28\%); LGO5 (14 of 55, 25\%); LG06 (19 of 104, 18\%); and LG08 (25 of 106, 24\%). Although the proportion of identical FL env sequences differed significantly across compartments, we also detected a modification of effect between participant and compartment $(P<0.001)$. In other

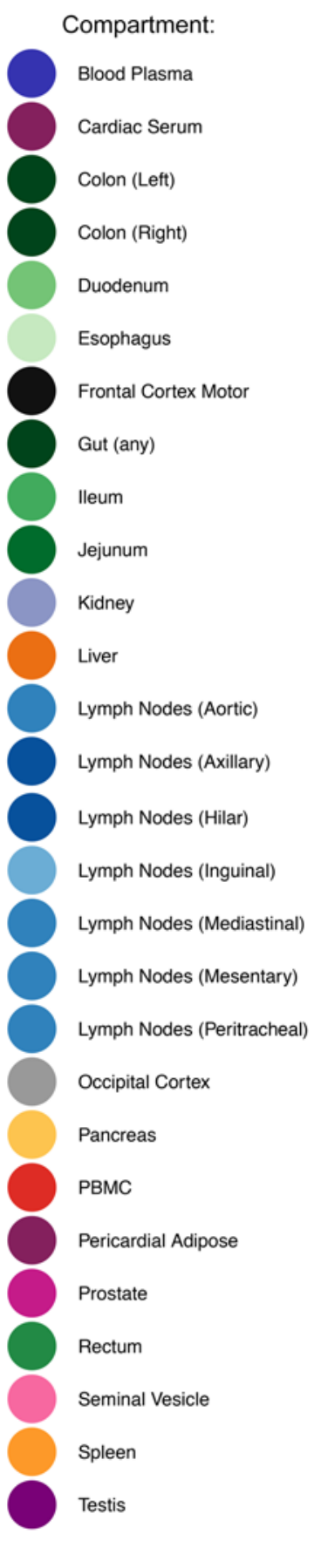

Figure 4. ML phylogenies and clonal populations (FL envelope) for 2 participants who either stopped ART or remained virally suppressed on ART. IQ-TREE (97) was used to estimate phylogenies for the FL HIV env sequences obtained from premortem blood plasma and tissues and from PBMCs collected during rapid autopsy. (A) ML phylogeny for participant LG01, who stopped therapy. (B) ML phylogeny for participant LG03, who remained virally suppressed. Tips are colored by compartment as in the legend. The size and distribution of nearly identical FL env populations ( $99 \%$ identical, populations of at least 3 identical proviruses) for each participant are presented in the middle of each tree. Colors represent the tissues described in the key. For LG01, nearly identical FL env populations including HIV RNA viruses sampled in blood plasma during viral rebound are marked with an asterisk. See also Supplemental Figure 4 for data on the 4 remaining participants. words, the differences across compartments varied according to participant, with no consistent clonality trends observed across tissues with or without sustained viral suppression.

Viral tropism and repopulation. HIV DNA populations in antemortem PBMCs and postmortem tissues were predominantly X4 tropic, based on genotropism analysis for 4 participants (LG01, 84\%; LG03, 75.5\%; LG05, 78.2\%; and LG06, 54.5\%), whereas X4-tropic proviruses accounted for only $14.9 \%$ and $6.6 \%$ of sequences obtained from LG04 and LG08, respectively (Supplemental Table 2 and Supplemental Figure 5). Next, we evaluated tissue repopulation during rebounding viremia in the 2 participants who voluntarily decided to interrupt their therapy. 


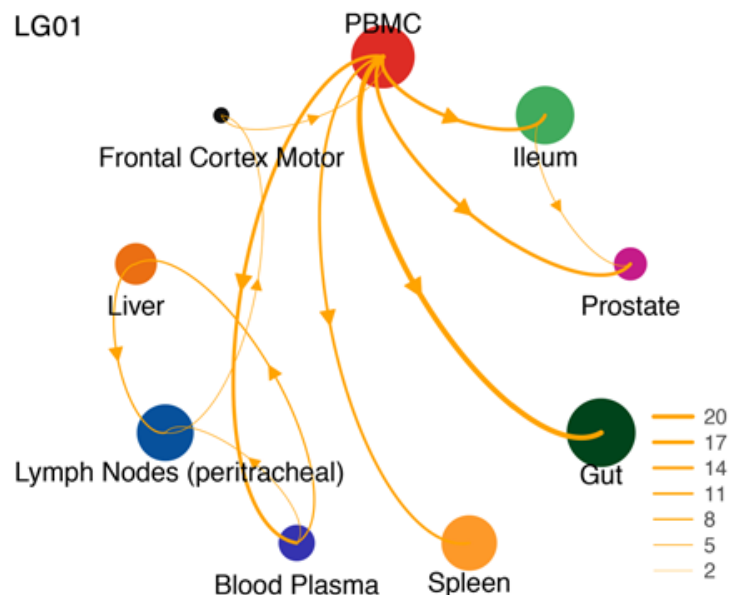

LG04

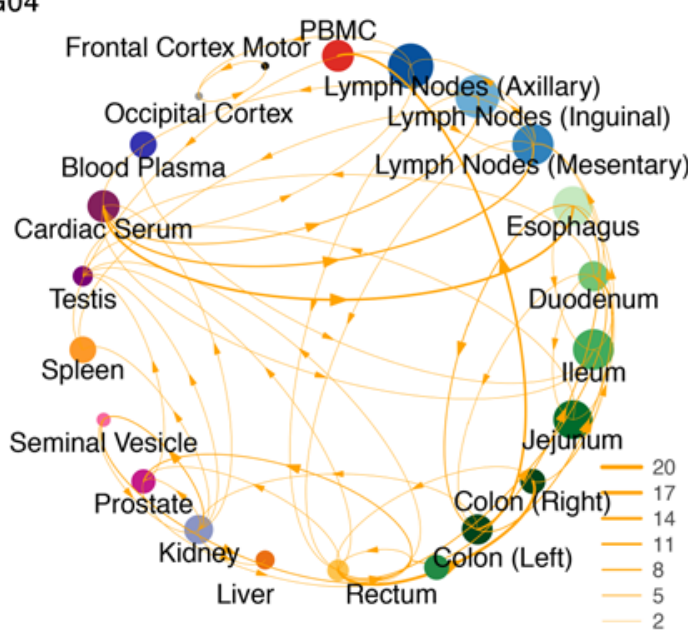

LG06

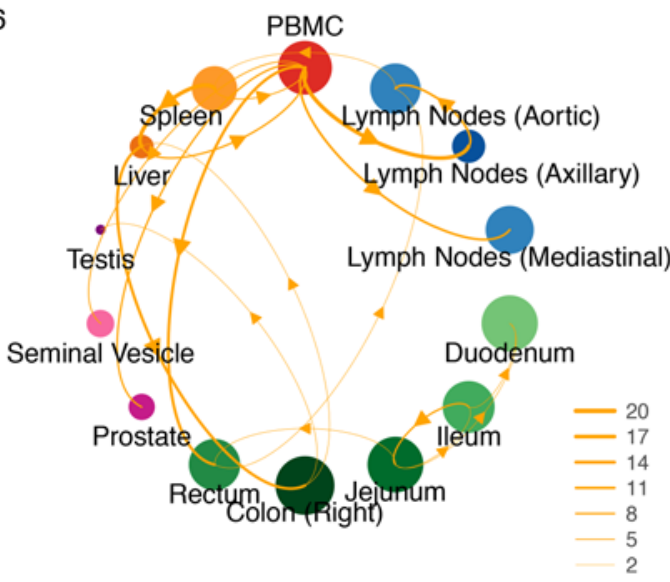

LG03

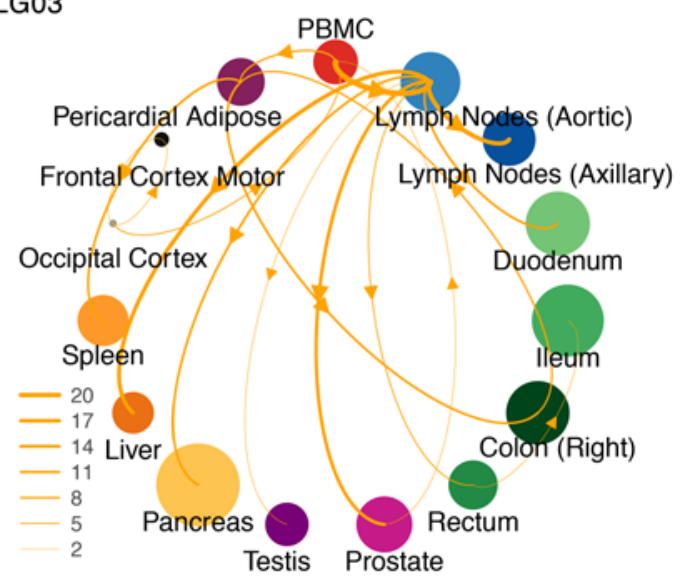

LG05

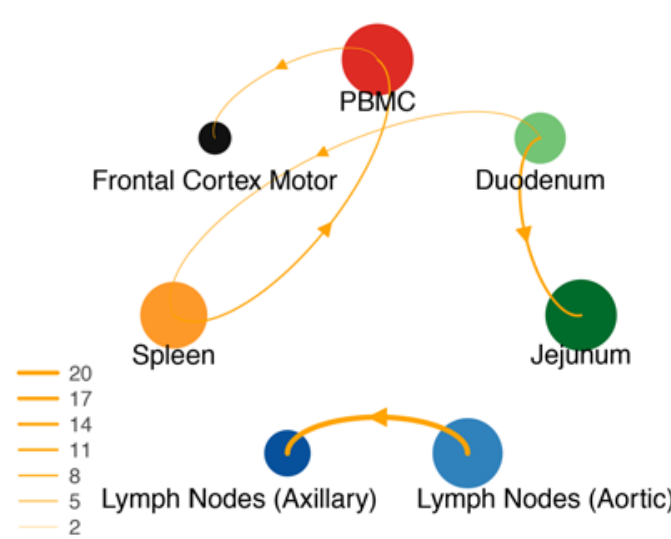

LG08
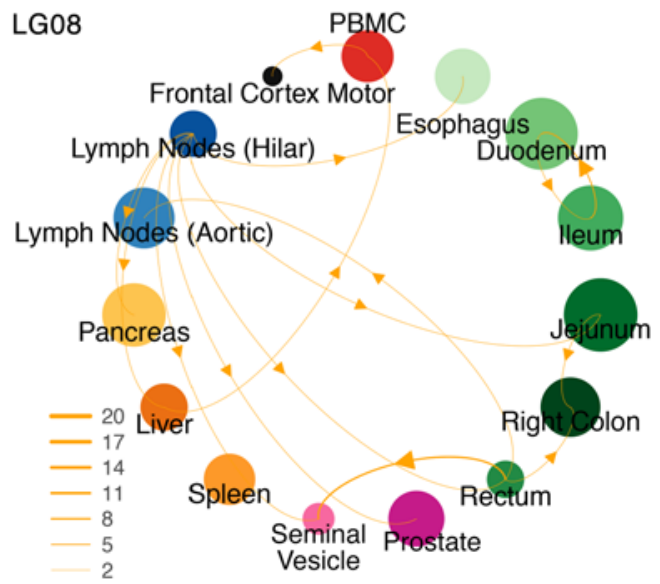

Figure 5. Lineage dispersal events between compartments. The circle size is proportional to HIV DNA levels (or RNA for plasma) in each compartment. The thickness of the arrows corresponds to the average number of inferred migration events between compartments. Only transition events between locations for which the adjusted BF was 3 or higher (at least positive evidence) are shown.

For participant LG04, who stopped ART more than 2 months before his death, his last HIV RNA level in blood plasma before death was 48,000 copies/mL. The rebounding HIV RNA was $100 \%$ intact in $e n v$ and nearly clonal (10 of 10 intact, nearly iden- tical [ $\geq 99 \%$ identity] FL env proviruses), and $80 \%$ of the variants were R5 tropic (Supplemental Figures 4 and 5). Interestingly, we also found identical R5-tropic FL env sequences in the HIV DNA population in antemortem PBMCs (17 of 23 sequences) and across 


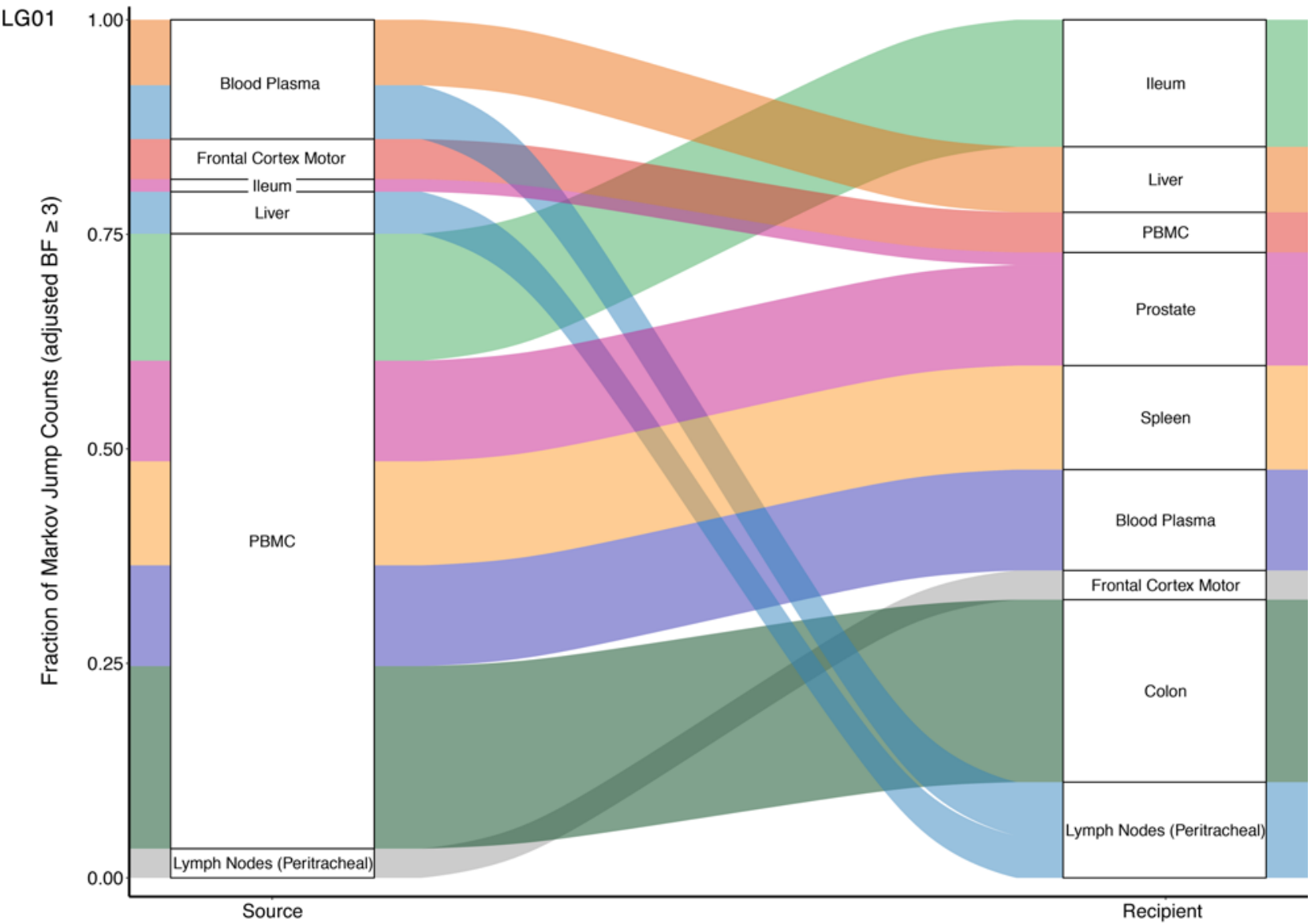

B

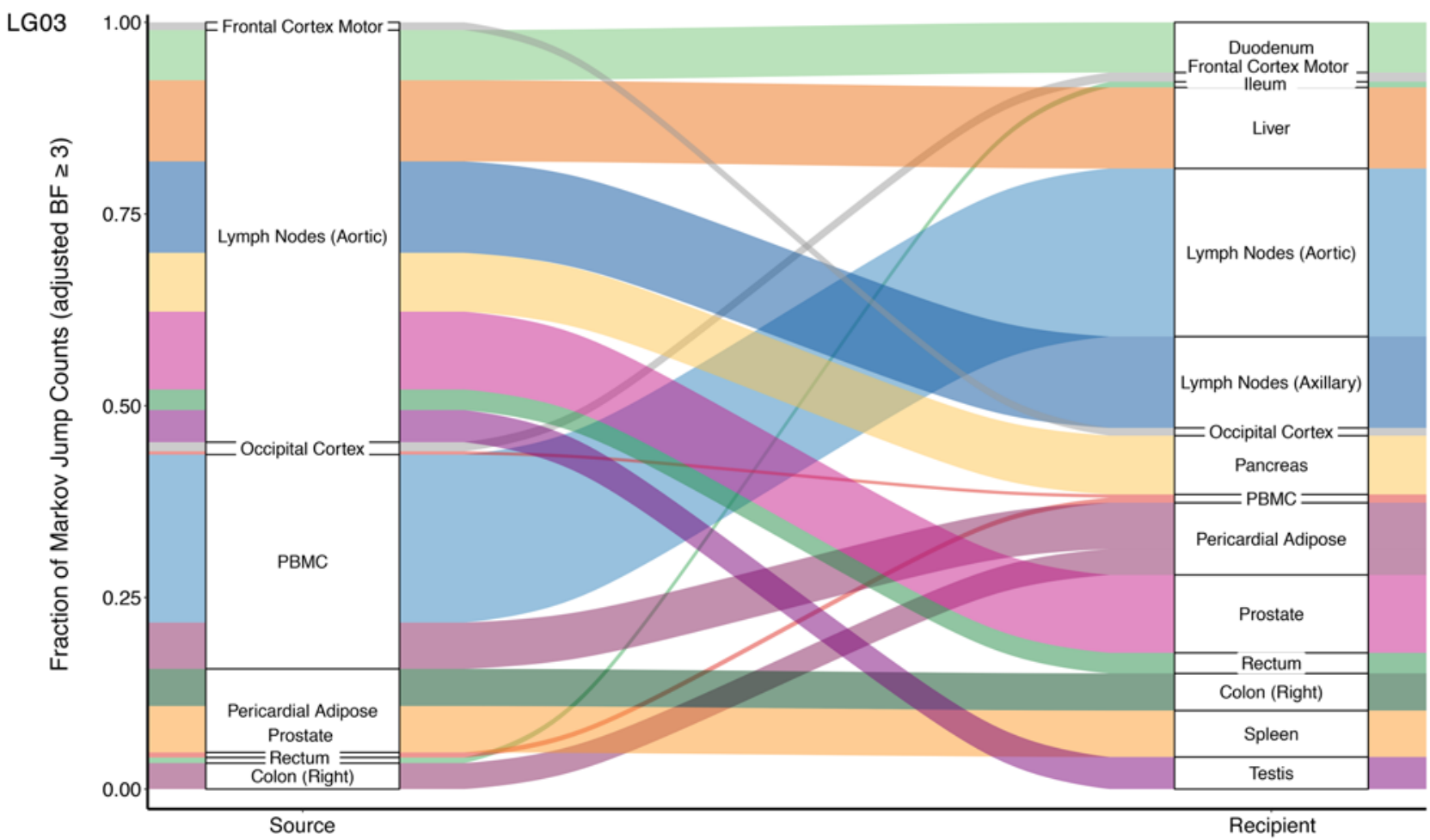

Figure 6. Proportion of supported transition events between compartments for 2 participants who either stopped ART or remained virally suppressed on ART. (A) Data for the participant who stopped ART. (B) Data for the participant who remained virally suppressed on ART. Sankey plot shows the proportion of transition events between locations for which the adjusted BF was 3 or higher (at least positive evidence). The adjusted BF support for each transition type is given next to the corresponding color. The source locations are depicted on the left side of the plots and the destination locations on the right side. See also Supplemental Figure 6. 
almost all other sampled tissues collected postmortem, including those from lymph nodes, the genital tract, and gut tissues (Supplemental Figure 4). Although we cannot be sure of the origin of the rebounding R5-tropic population because antemortem samples were only available for PBMCs and blood plasma, this observation strongly suggests that many tissue reservoirs were repopulated by R5-tropic virus during rebound.

Participant LG01 stopped ART 53 days before his death, and his last HIV RNA level in blood plasma before death was 280 copies/mL. Again, the rebounding HIV RNA population was $100 \%$ intact (17 of 17) and predominantly clonal (13 of 17 intact, nearly identical [ $\geq 99 \%$ identity] FL env). Phylogenetic analyses also revealed a large, nearly identical FL env population of $20 \mathrm{X} 4$-tropic proviruses in postmortem gut $(n=5)$, PBMCs $(n=4)$, prostate $(n=$ $3)$, and blood plasma $(n=8)$ (Figure 4 and Supplemental Figure 5).

Viral dispersal and repopulation. As expected, all sequences clustered by participant when combined in a single phylogeny (Supplemental Figure 3), and using phylogenetic trait (i.e., anatomical site) association measures, we found evidence of viral compartmentalization by anatomical site in all 6 participants $(P$ $<0.01$ ) (Supplemental Table 3). Discrete phylogeographic models showed various patterns of viral dispersal (Figures 4 and 5; only transition events between locations for which the adjusted Bayes factor $[\mathrm{BF}]$ of 3 or higher are shown).

Among participants who remained virally suppressed (LG03, LG05, LG06, and LG08), the majority of transition events originated from lymph nodes and gut tissues (Figures 5 and 6 and Supplemental Figure 6). We also found strong evidence for viral dispersal from lymph nodes to prostate in participant LGO3 (BF = 568) and from rectum to seminal vesicles in participant LG08 (adjusted $\mathrm{BF}=74$ ). Interestingly, there was also positive support for viral transitions from PBMCs to frontal lobes in participants LG05 (adjusted BF $=11$ ) and LG08 (adjusted BF = 5.5).

Among the participants who stopped ART (LG01 and LG04), we sequenced FL env from HIV RNA from blood plasma collected antemortem during viral rebound (HIV RNA levels of 13,500 copies/mL and 48,000 copies/mL, respectively). For participant LG01, we found strong evidence of viral dispersal from PBMCs for all transition events (BFs $\geq 20$ ) toward gut sites, prostate, spleen, and blood plasma (adjusted BFs $=449.7,62,27$, and 22.7, respectively). There was also good support $(\mathrm{BFs} \geq 10$ ) for transition events from blood plasma toward lymph nodes (adjusted BF = 16.6) and liver (adjusted $\mathrm{BF}=10.3$ ) and from liver toward lymph nodes (adjusted $\mathrm{BF}=11.8$ ). We also found positive support for viral migration from the frontal lobe toward PBMCs (adjusted BF $=3.9$ ) and from lymph nodes toward the frontal lobe (adjusted BF $=10.4$ ) (Figures 5 and 6). For participant LG04, we found that 9 sources of viral migration were strongly supported (BFs $\geq 20$ ), including migration from blood plasma toward gut tissues and intense viral exchanges between the sampled gut sites (Figure 5 and Supplemental Figure 6). Interestingly for participant LGO4, we also found evidence of bidirectional transition events within the CNS, from the occipital lobe toward the frontal lobe (adjusted BF $=25.4$ ) and from the frontal lobe toward the occipital lobe (adjusted BF = 10.1). The lack of dominance of particular transition types shows that a diverse set of anatomical compartments can act as the source of reservoir virus.
Predictors of viral dispersal. We next used a generalized linear model (GLM) extension of the discrete phylogeographic model to determine whether the number of proviruses, viral diversity, proportion of X4-tropic viruses, and viral divergence between compartments are associated with potential dispersal rates. We first assessed collinearity between all the factors evaluated and found that all correlation coefficients were less than 0.8 . For participants LG03, LG04, and LG06, the model consistently revealed strong evidence that viral migration was associated with limited divergence between the source and recipient compartments (respective adjusted BFs $=389.7,54.4$, and 1690.4; Supplemental Figure 7). For LGO3 and LGO4, there was also evidence of an association between the transition rate and greater viral diversity in the recipient compartment (BFs $=373.9$ and $\geq 1.5 \times 10^{4}$, respectively). Finally, for LG03 only, we noted a positive association between a lower proportion of $\mathrm{X} 4$-tropic viruses in the source compartment and viral dispersal $(\mathrm{BF}=5.8)$. For participants LG01, LG05, and LG08, we found no evidence that any of the tested factors were associated with viral dispersal (Supplemental Figure 7).

\section{Discussion}

Understanding how HIV populates tissues throughout the human body is crucial for the development of strategies to clear or otherwise lock down these reservoirs (47-49). Such investigations have been hampered by limitations in technology and access to fresh sampling of tissues throughout the body. This study used samples and data from 6 participants in a perimortem cohort (Last Gift) of altruistic PWH who provided blood samples before they died and their whole bodies immediately after death. Given the freshness of samples, viral populations probably remained intact, so virologic data were generated and analyzed using state-of-the-art methods. The main findings of this study were as follows: (a) large, clonal, intact HIV RNA populations emerged in blood plasma after cessation of ART and repopulated tissues throughout the body; (b) multiple sites could act as hubs for dissemination of HIV within the host, predominately in the blood, gut, and lymph nodes; (c) viral exchanges occurred within CNS areas and between the CNS and blood; (d) viral dynamics were associated with low HIV divergence between sites and high HIV diversity at the recipient site. Together, these data provide insights into how HIV populates reservoirs across the human body.

Previous studies showed that lymphoid tissues (e.g., spleen, thymus, lymph nodes, gut-associated lymphoid tissue) are the sites with the highest level of viral replication during active infection, and HIV DNA can be readily cultured from lymphoid tissues after years of treatment (5). HIV-infected cells have also been found in the CNS, lungs, kidneys, liver, adipose tissue, genitourinary tract, and bone marrow $(5,50-53)$. In agreement with these observations, we found intact FL HIV-1 env throughout the 28 anatomic compartments sampled from 6 participants of the Last Gift cohort. It was interesting that participant LGO3 had very high levels of HIV DNA in his pancreas (391.5 gag copies/106 cells [329.2-453.7]), which is also where his terminal cancer occurred. Since T cells infiltrate tumor microenvironments, it is possible that the resident tumor attracted HIV-infected T cells (54). More tumor tissues will need to be evaluated to confirm this observation. 
Consistent with other reports that investigated blood from PWH (55-57), our study found various proportions of identical FL env sequences in PBMCs (varying from 19\% [CI 95\%: 4\%-46\%] to $81 \%$ [CI 95\%: 54\%-96\%] for LG08 and LG01, respectively). We also found identical FL env sequences in various tissues (e.g., ileum and jejunum for LGO3 and LG04) and across multiple tissues. This observation could be explained either by infection of susceptible cells at multiple sites by monoclonal HIV RNA, as seen in LGO4 during rebound viremia, or infection of cells that clonally expanded at one site and then migrated to a different site. These scenarios are not mutually exclusive. Although identical env sequences may reflect cellular proliferation, without integration site analysis, we cannot determine whether identical env sequences are derived from cellular clonal expansion (58). Future integration site analyses are needed to confirm this possibility, as illustrated by Patro et al. (59).

With regard to viral migration, this study found that not only blood but also mainly gut and lymphoid tissues can act as sources for the dissemination of HIV, which is consistent with previous studies, suggesting that both of these tissues are major HIV reservoirs during ART (60-65). Interestingly, we also observed that genital tract tissues (i.e., prostate, seminal vesicles, testis) were potential sources of viral dispersal to other tissues. Although limited by the number of intact FL env proviruses recovered from the frontal and occipital lobes (5 of 6 participants), this study shows that viral migration occurred within the CNS (LG03 and LGO4), between blood and the CNS (LG01, LG03, LG05, and LG08), and from the lymph nodes toward the CNS (LG01).

Assessing the factors that may contribute to the viral dynamics within and across compartments is likely to assist in developing strategies targeting the reservoirs; therefore, we used a GLM model to evaluate the role of viral characteristics (e.g., viral diversity, proportion of $\mathrm{X} 4$-tropic viruses, and viral divergence between compartments) as potential drivers of viral dispersal. Although these findings were limited to 6 participants and only involved viral population characteristics, we showed that limited viral divergence between compartments was strongly associated with viral dispersal in 3 of 6 participants. We also showed that viral dispersal was associated with greater viral diversity in the recipient (2 of 6 participants) and a lower proportion of X4-tropic viruses in the source ( 1 of 6 participants). Although these results remain limited and require validation in a larger cohort, they suggest that HIV dynamics across the body are also influenced by molecular characteristics of the HIV populations. Further analyses evaluating pharmacological and immunological factors in the HIV microenvironment would be of interest.

Some PWH toward the end of their lives wanted to stop ART before they died. This unique situation allowed us to observe viral rebound and tissue repopulation in 2 participants with rebound viremia who voluntarily interrupted their ART 53 and 70 days before death. Consistent with the study by De Scheerder et al. (40), our phylogenetic and statistical analyses show that rebounding viral populations can originate from various anatomical compartments with no prominent source of rebound. Also consistent with previous studies (66), we showed that rebounding HIV RNA populations were composed of intact, nearly identical FL $e n v$ sequences, and thus we found that HIV RNA populations were more homogenous than HIV DNA populations in tissues in the 2 participants who stopped therapy $(P=0.025)$. Interestingly, when participant LG04 stopped ART, he had an asymptomatic rebound of a large, intact, nearly identical R5-tropic HIV RNA population (FLenv) in the blood. This population was identical to a HIV DNA population seen in PBMCs antemortem. After death, we found identical FL env sequences in tissues throughout the body including in lymph nodes, the genital tract, and gut tissues. Similarly, participant LG01 had rebound viremia with an intact, nearly identical X4-tropic population, and we found identical FL env sequences in the gut, PBMCs, and prostate. Although not conclusive, because we did not sample tissues before the participants stopped ART, this strongly suggests that the rebounding HIV population in blood plasma was the source of viral replenishment in tissue reservoirs. It also supports the notion that preventing HIV rebound in the blood may allow for ART-free remission, like what was observed in Timothy Brown and the London patient, when their previously HIV-susceptible circulating CD $4^{+}$ $\mathrm{T}$ cells were replaced by bone marrow transplantation of $\mathrm{CD} 4^{+} \mathrm{T}$ cells that were no longer susceptible to HIV infection because of the CCR5 $32 \Delta$ deletion $(67,68)$.

Our study has a number of limitations. The main limitation is the small number of participants and, especially, the fact that only 2 participants stopped ART. Nonetheless, it allowed us to observe the population of tissue reservoirs from the blood during rebound viremia. Another limitation is that the participants were all in the process of dying, which may limit the generalizability to healthy PWH. Also, this study, like others $(69,70)$, focused on the HIV env gene, which has the greatest amount of molecular diversity and evolution of all coding regions (71-76), but we acknowledge that by sequencing only the $e n v$ region, we may have incorrectly inferred that some viruses intact in the env coding region were replication competent, when they may have had defects in other genome regions. Further, the study found many identical HIV DNA env single genomes, consistent with previous reports (77-80), but we did not directly assess cellular clonal expansion, which is thought to be an important mechanism for HIV persistence (81-83). However, others have shown that multiple identical env sequences in proviruses provide a strong indication for clonal expansion $(84,85)$. Sequencing a near-FL proviral genome (86-88) would increase the sensitivity of the analyses of intactness and clonality, but these approaches require a high cellular input that may limit the ability to explore reservoirs such as the CNS. Therefore, we are confident that our approach provided a good surrogate for the extensive analysis of HIV reservoirs. Further, we did not perform phenotypic testing of CCR5 tropism, and every participant in our study had $\mathrm{X} 4$-tropic virus by genotypic analysis. This is probably because participants were infected for a long time before enrollment in the study (89) and emphasizes that individuals with longstanding HIV infection are unlikely to benefit from measures focusing only on CCR5-tropic viruses, as was the case for the $2 \mathrm{PWH}$ who received CCR5-mutated bone marrow transplants and have been cured of HIV so far $(68,90)$. Finally, we cannot exclude the possibility of blood $\mathrm{T}$ cell contamination in tissues obtained during autopsy. This is likely to have a small impact on our analysis, given the small size of capillaries compared with overall 
tissue mass and the settling of blood in tissues, i.e., livor mortis. Although we cannot completely rule out such contamination, our sequence analyses showed viral compartmentalization for all participants, which suggested that possible blood contamination did not significantly affect our analyses.

In conclusion, this study leveraged the unique Last Gift cohort of participants who continued or discontinued ART before dying, after which multiple tissues were rapidly collected at autopsy. From these samples, the study reconstructed the spatial dynamics of HIV across the body and found that multiple body compartments, especially the gut and lymphoid tissues, can act as hubs for dissemination of HIV and that blood is the likely conduit for dispersal. This study also uniquely clarified the HIV dynamics within the CNS and across the blood-brain barrier. Importantly for cure efforts, our analyses strongly indicated that rebounding virus was derived from circulating reservoirs and that rebounding virus quickly populated deep tissue reservoirs throughout the body via the blood. Given the extent of intact FL env HIV populations throughout the body, it is likely that any of the tissue reservoirs could facilitate reseeding of virus to the blood and thus repopulate tissue reservoirs. These findings might have consequences for treatment interruption studies, although the clinical significance is unclear (91). Thus, for cure efforts aimed at eradication, all such reservoirs would need to be cleared. Alternatively, functional cure efforts could focus on making circulating $\mathrm{CD} 4^{+} \mathrm{T}$ cells impervious to infection to prevent rebound viremia and subsequent viral dispersal and reseeding, although this approach would not address local viral reactivation and damage.

\section{Methods}

\section{Study cohort and sampling}

PBMCs and tissue samples were obtained from the first $6 \mathrm{PWH}$ enrolled in the Last Gift cohort $(92,93)$. Study participants were followed closely near the end of their lives (approximatively every other week, with closer intervals as death approached). During these visits, participants provided: (a) detailed clinical and sociodemographic information before their death (use of ART, chemotherapy and other therapies, surgical procedures, coinfections, etc.); (b) blood samples while they were alive; and (c) their entire bodies after they died for a rapid autopsy. Clinical characteristics of the study participants are summarized in Supplemental Table 1 and Supplemental Figure 2.

\section{Rapid autopsy}

The Last Gift rapid autopsy protocol was designed to collect tissues within 6 hours of death to minimize postmortem tissue degradation. At the time of death, the body was rapidly transported to the UCSD morgue, where the team performed a complete autopsy of all organs to obtain tissue samples, which were either formalin-fixed and paraffin-embedded for histological analysis or snap-frozen in liquid nitrogen. Fluids collected included CSF and blood drawn from cardiac puncture.

\section{HIV DNA quantification and sequencing}

DNA extraction, purification, and quantification. Genomic DNA was extracted from 5 million PBMCs and snap-frozen tissues using a
QIAamp DNA Mini Kit (QIAGEN, catalog 51306) according to the manufacturer's protocol. After extraction, precipitation was performed to concentrate DNA. Concentrations of DNA were determined using NanoDrop One (Thermo Fisher Scientific). Levels of extracted HIV DNA were quantified by droplet digital PCR (ddPCR) using the Bio-Rad QX200 Droplet Reader (94). Copy numbers were calculated as the mean of 3 replicate PCR measurements and normalized to 1 million cells, as determined by RPP30 assay (total cell count) $(94,95)$.

RNA extraction and quantification. RNA was extracted from blood plasma by layering $500-700 \mu \mathrm{L}$ plasma on top of $200 \mu \mathrm{L} 20 \%$ sterile filtered sucrose solution. Samples were spun at 23,500 $\times g$ for 1 hour at $4^{\circ} \mathrm{C}$ to pellet the virus. Supernatant was removed and the pellet resuspended in $140 \mu \mathrm{L}$ PBS. RNA was extracted using a QIAamp Viral RNA Mini Kit (QIAGEN, catalog 52904) according to the manufacturer's recommendation. cDNA from HIV RNA was generated using the OneStep RT-ddPCR Advanced Kit for Probes (Bio-Rad, catalog 186-4021), and levels were quantified by ddPCR using the Bio-Rad QX200 Droplet Reader. Copy numbers were calculated as the mean of 3 replicates.

Nested PCR. To amplify single-genome FL env, DNA extracted from antemortem PBMCs and postmortem tissues was diluted using ddPCR quantification data. This limited dilution PCR reaction can prevent PCR recombination and ambiguous base calls and allow the amplification of viral single genomes $(87,96)$. For HIV RNA in blood plasma, cDNA was generated from RNA using the SuperScript III First Strand Synthesis System (Thermo Fisher Scientific, catalog 18080-051). Template cDNA and HIV DNA extracted from tissues were diluted until approximately $30 \%$ of the second-round reactions were positive for the correctly sized amplification product. Primers used for the first round were as follows: $5^{\prime}$-FENVouter (forward) TTAGGCATCTCCTATGGCAGGAA and 3'-RENVouter (reverse) TCTTAAAGGTACCTGAGGTCTGACTGG. First-round PCRs were performed using the Advantage 2 PCR Kit (Takara, catalog 639206) following the manufacturer's recommendations and 10× SA buffer (Takara, catalog 639206). Cycling conditions were as follows: $95^{\circ} \mathrm{C}$ for 1 minute, 35 cycles of $95^{\circ} \mathrm{C}$ for 15 seconds, $57^{\circ} \mathrm{C}$ for 30 seconds, $68^{\circ} \mathrm{C}$ for 3 minutes, with a final extension at $68^{\circ} \mathrm{C}$ for 10 minutes. The second-round PCRs were done using $5^{\prime}$-FENVinner (forward): GAGCAGAAGACAGTGGCAATGA and 3'RENVinner (reverse): CCACTTGCCACCCATBTTATAGCA. The cycling conditions were as follows: $95^{\circ} \mathrm{C}$ for 1 minute, 30 cycles of $95^{\circ} \mathrm{C}$ for 15 seconds, $64^{\circ} \mathrm{C}$ for 30 seconds, and $68^{\circ} \mathrm{C}$ for 3 minutes, with a final extension at $68^{\circ} \mathrm{C}$ for 10 minutes. PCR cleanups were performed on the second-round reaction products using a QIAquick PCR Purification Kit (QIAGEN, catalog 28106). DNA was quantified using a Qubit dsDNA HS Assay Kit (Invitrogen, Thermo Fisher Scientific, catalog Q32854). Quality and integrity were measured using Genomic DNA ScreenTape (Agilent Technologies, catalog 5067-5365) in combination with the 2200 TapeStation System (Agilent Technologies, Genomic DNA Reagents, catalog 5067-5366).

Nextera XT library preparation. Single-genome amplicons were prepared for deep sequencing using the Nextera XT DNA Library Preparation Kit (Illumina, FC-131-1096) with indexing of 96 samples per run (Nextera XT Index Kit, set A FC-131-2001) according to the manufacturer's protocols.

Assembly of FL HIV env proviruses. We used a custom-designed pipeline to recover FL env HIV sequences from the paired-end reads. The pipeline included a preliminary step of quality control, 
which involved trimming reads for PHRED quality above or equal to 30 and removal of Illumina adapters. Next, overlapping identical paired forward and reverse reads were merged and premapped to the HXB2 reference genome. Cleaned reads were remapped to the de novo assembled near-FL env sequence before generation of the final consensus sequence. The minimum acceptable coverage was set to 10,000 reads. To identify mixtures (i.e., suggesting multiple amplified HIV templates), all generated FL env contigs were screened. Mixtures were identified on the basis of read coverage and variant calling. Contigs with evidence of SNPs with a frequency of greater than $1 \%$ were considered mixtures and excluded from further analyses.

Test for cross-contamination. A maximum likelihood (ML) phylogeny including all sequences from the 4 participants was estimated using IQ-TREE (http://www.iqtree.org/) with the general time-reversible (GTR) substitution model (97) to test for contamination, which would show as intermixed clustering of taxa between participants.

Identification of defective or hypermutant sequences. FL envelopes containing large deletions ( $>100 \mathrm{bp}$ ) were considered defective (86, 98). Deleterious stop codons were identified using the Gene Cutter tool (Los Alamos HIV Database; https://www.hiv.lanl.gov/content/ sequence/GENE_CUTTER/cutter.html). Any contigs containing a stop codon were considered defective. APOBEC-induced (apolipoprotein $\underline{B}$ mRNA-editing enzyme, catalytic polypeptide-like-induced) G-A hypermutations were identified using the Los Alamos HIV Database Hypermut 2 program (https://www.hiv.lanl.gov/content/ sequence/HYPERMUT/hypermut.html) and the participant's consensus sequence $(99,100)$. Proviruses with a number of mutations significantly higher than those in the participant's consensus $(P<$ 0.05 , Fisher's exact test) were considered hypermutant and were not included in the downstream analyses described below.

\section{Sequence analyses}

Identification of identical FL env sequences. To determine the sequences that were greater than or equal to $99 \%$ or $100 \%$ genetically identical, we used the ElimDupes tool from the Los Alamos HIV database, with a genetic identity threshold of $99 \%$ or greater or $100 \%$ as the analysis parameter (101). A sequence was classified as identical if it was a $100 \%$ match against another sequence sampled from the same participant. Once identified, the proportion of identical and nearly identical sequences was calculated by dividing the total number of sequences classified as identical/nearly identical for each participant or compartment by the total number of sequences for that group.

Diversity and divergence. Viral diversity was defined as the average pairwise genetic distance between sequences from a compartment using the TN93 correction for multiple hits (46). Viral divergence was assessed by computing the mean pairwise distance (TN93) between viral populations sampled across anatomical sites.

Coreceptor tropism. Viral tropism of each variant was inferred from the V3 amino acid sequence using Geno2pheno (https://coreceptor. geno2pheno.org/) (102). We applied a conservative $10 \%$ false-positive rate threshold for coreceptor CXCR4 usage on the basis of the European Consensus Group's recommendation on clinical management of HIV-1 tropism testing.

Viral migration. Before evaluating the within-host migration processes, the level of spatial structure was quantified using the Simmonds association index (AI) implemented in BaTS, version 1.0 (103). We used the BEAST software package, version 1.10 (https:// beast.community/) (104) for all evolutionary analysis. All sequences were considered isochronous, i.e., branch lengths were estimated in units of substitutions per site. For this, a strict molecular clock was specified, and the clock rate was fixed to 1 . The substitution process was described with a HKY $+\Gamma$ model $(105,106)$, and a constant population size was assumed. Discrete trait analyses were performed using the asymmetric diffusion model $(107,108)$. To identify the subset of migration rates that were most informative to reconstruct the dispersal history, we used a model averaging procedure (Bayesian stochastic search variable selection [BSSVS]) (107). BF support for all possible types of location exchanges was calculated using SpreaD3 (https://rega.kuleuven.be/cev/ecv/software/SpreaD3) (109). BFs between 3 and 20, between 20 and 150, and above 150 were considered positive, strong, and decisive support, respectively (110). Estimates of the posterior expected number of migration events between all pairs of locations (Markov jumps) were computed using stochastic mapping techniques $(105,111)$. To identify individual-level differences in within-host viral diffusion patterns, the trait analyses were performed on a patient-specific basis.

To investigate whether support for migration links followed from the relative abundance of the involved trait states, analyses were repeated while randomly permuting the compartment states between tips during the Markov chain Monte Carlo (MCMC) sampling (112), a technique analogous to the tip-date randomization procedure for testing the significance of the temporal signal $(113,114)$. Indeed, if the support for a particular migration rate persisted after randomizing the tip-to-location assignments, one cannot rule out the possibility that the support is due to sampling intensity differences. Furthermore, of the migration links that passed the above filter (good BF support in the "as is" analysis and poor BF support in the "tip-state-swap" analysis), only those that remained significant after accounting for the sampling heterogeneity were taken into account to further reduce the false-positive rate. To this end, the inclusion frequencies of the "tip-state-swap" analysis instead of those of the "as is" analysis were treated as the prior inclusion probabilities when recomputing the BF support, hereafter referred to as the adjusted BF. Only the results with an adjusted BF with positive support $(\mathrm{BFs} \geq 3$ ) are further discussed in this study. This approach was also used to investigate the sensitivity of the Simmonds AI to the sampling heterogeneity.

Multiple MCMC chains were run for a sufficient length of time to ensure convergence and adequate mixing (effective sample size [ESS] >200), which was inspected using Tracer, version 1.7 (https://beast. community/tracer) (115). The chains were sampled every 500,000 generations and combined after removal of the burn-in. Maximum clade credibility (MCC) trees were obtained with TreeAnnotator, version 1.10 (https://beast.community/programs) (104).

Identification of predictors of within-host spread. The GLM extension of the discrete trait model implemented in BEAST (116) was used to investigate the relevance of potential explanatory variables (predictors) to explaining the dispersal across body compartments. The following variables were included: (a) the number of FL intact env provirus recovered in each compartment to control for sampling bias effects (116); (b) the level of HIV DNA (ddPCR gag copy/10 6 cells for all antemortem PBMC and postmortem tissue samples) or level of HIV RNA for antemortem blood plasma samples (number of copies/ $\mu \mathrm{L}$ ); (c) viral diversity, estimated using the average pairwise genetic distance between each sequence; and (d) the proportion of X4-tropic 
provirus in each compartment. For each of these variables, the values in the compartment source and recipient were considered. We also included a matrix of pairwise measurements of TN93 distance between compartments (i.e., viral divergence). Colinearity between the variables included in the GLM model that was evaluated. When 2 variables showed a collinearity coefficient of 0.8 or higher, the analysis was repeated with only 1 variable in the model.

\section{Statistics}

Multivariable logistic regression was carried out in $\mathrm{R}$, version 3.6.1, applying the function GLM and binomial link function, and was used to compare the proportion of sequences that were intact and identical (clonal). The independent variables in these analyses were participant and anatomical location. For the continuous average pairwise distance (diversity) outcome, multiple linear regression was used, with assumptions of constant variance and normality of residuals checked and met. Mixed models for both binary and continuous outcomes were analyzed with glmer and lmer from the lme4 R library. These mixed models used participant as the grouping factor and included a random term for either intercept alone, or intercept and compartment. Given the sparse nature of the data, all mixed models had difficulty converging with poor model fits. As such, the results and $P$ values of these models were not reliable, and so the results are not presented. Given the large number of tissue compartments, the sparse sampling across participants, and the expected effect modification by participant and compartment, comparisons across specific compartments were not informative.

\section{Study approval}

All study participants were at least 18 years of age and provided written, informed consent. This study was approved by the UCSD Office of Human Research Protections Program (protocol 160563). One of the participants exercised his legal right-to-die option in the state of California (117). No government funds were used as part of the option.

\section{Author contributions}

AC, SG, CI, MP, BV, SD, SAR, and DMS conceived and designed the experiments. AC, CI, and MP performed the experiments. AC, SG, DMS, BV, SD, and TES analyzed the data. Enrolled participants. SG, DMS, and SAR enrolled the participants. AC, SG, DMS, MFDO, BV, SD, SAR, and TS wrote and revised the manuscript.

\section{Acknowledgments}

We are very grateful to the Last Gift participants and their families. We would like to acknowledge the investigators and team members of the Center for AIDS Research (CFAR) Genomic and Sequencing and Translational Virology Cores. We would like to acknowledge Gemma Caballero, Laura Layman, and Brianna Scott for their technical assistance. We would also like to acknowledge Steven Hendrix and Susanna Concha Garcia for their assistance with participant enrollment, and Susan Little and Matt Strain for the very helpful discussions. AC received funding from the NIH (AI131971-01 and AI036214); the UCSD CFAR; a NIH-funded program; and the San Diego CFAR Translational Virology (TV) Core. SG acknowledges funding from the NIH (HD094646, AIO27763, AI134295, and AI68636). DMS received from the NIH (PO1 AI31385 and P30 AI036214). MP was supported by the San Diego Veterans Medical Research Foundation (VMRF). SD was and BV is supported by a postdoctoral fellowship from the Fonds voor Wetenschappelijk Onderzoek (FWO) of Belgium. SD is currently supported by the Fonds National de la Recherche Scientifique (FNRS) of Belgium.

Address correspondence to: Antoine Chaillon, Department of Medicine, University of California San Diego, 9500 Gilman Drive MC 0679, La Jolla, California 92093-0679, USA. Phone: 858.552.8585 ext. 2673; Email: achaillon@ucsd.edu.
1. Whitney JB, et al. Rapid seeding of the viral reservoir prior to SIV viraemia in rhesus monkeys. Nature. 2014;512(7512):74-77.

2. Cohen MS, Shaw GM, McMichael AJ, Haynes BF. Acute HIV-1 Infection. N Engl JMed. 2011;364(20):1943-1954.

3. Mzingwane ML, Tiemessen CT. Mechanisms of HIV persistence in HIV reservoirs. Rev Med Virol. 2017;27(2):e1924.

4. Kahn JO, Walker BD. Acute human immunodeficiency virus type 1 infection. N Engl JMed. 1998;339(1):33-39.

5. Wong JK, Yukl SA. Tissue reservoirs of HIV. Curr Opin HIV AIDS. 2016;11(4):362-370.

6. Yukl SA, JKW. In: Hope T, Stevenson M, Richman $\mathrm{D}$, eds. Anatomic compartments as a barrier to HIV cure. Encyclopedia of AIDS. New York: Springer; 2015:1-29.

7. Siliciano JD, et al. Long-term follow-up studies confirm the stability of the latent reservoir for HIV-1 in resting CD $4^{+} \mathrm{T}$ cells. Nat Med. 2003;9(6):727-728.

8. Finzi D, et al. Identification of a reservoir for HIV-1 in patients on highly active antiretroviral therapy. Science. 1997;278(5341):1295-1300.
9. Finzi D, et al. Latent infection of $\mathrm{CD} 4^{+} \mathrm{T}$ cells provides a mechanism for lifelong persistence of HIV-1, even in patients on effective combination therapy. Nat Med.1999;5(5):512-517.

10. Yukl SA, et al. The distribution of HIV DNA and RNA in cell subsets differs in gut and blood of HIV-positive patients on ART: implications for viral persistence. JInfect Dis. 2013;208(8):1212-1220.

11. van Marle G, Gill MJ, Kolodka D, McManus L, Grant T, Church DL. Compartmentalization of the gut viral reservoir in HIV-1 infected patients. Retrovirology. 2007;4:87.

12. Smith DM, Kingery JD, Wong JK, Ignacio CC, Richman DD, Little SJ. The prostate as a reservoir for HIV-1. AIDS. 2004;18(11):1600-1602.

13. Shen L, Siliciano RF. Viral reservoirs, residual viremia, and the potential of highly active antiretroviral therapy to eradicate HIV infection. J Allergy Clin Immunol. 2008;122(1):22-28.

14. Saksena NK, Haddad DN. Viral reservoirs an impediment to HAART: new strategies to eliminate HIV-1. Curr Drug Targets Infect Disord. 2003;3(2):179-206.

15. Lorenzo-Redondo R, et al. Persistent HIV-1 replication maintains the tissue reservoir during therapy. Nature. 2016;530(7588):51-56.

16. Katlama C, et al. Barriers to a cure for HIV: new ways to target and eradicate HIV-1 reservoirs. Lancet. 2013;381(9883):2109-2117.

17. Hong FF, Mellors JW. Changes in HIV reservoirs during long-term antiretroviral therapy. Curr Opin HIV AIDS. 2015;10(1):43-48.

18. Hodel F, Patxot M, Snäkä T, Ciuffi A. HIV-1 latent reservoir: size matters. Future Virol. 2016;11(12):785-794.

19. Sengupta S, Siliciano RF. Targeting the latent reservoir for HIV-1. Immunity. 2018;48(5):872-895.

20. Archin NM, Sung JM, Garrido C, Soriano-Sarabia N, Margolis DM. Eradicating HIV-1 infection: seeking to clear a persistent pathogen. Nat Rev Microbiol. 2014;12(11):750-764.

21. Archin NM, Margolis DM. Emerging strategies to deplete the HIV reservoir. Curr Opin Infect Dis. 2014;27(1):29-35.

22. Schwartz C, et al. On the way to find a cure: purging latent HIV-1 reservoirs. Biochem Pharmacol. 2017;146:10-22.

23. Kimata JT, Rice AP, Wang J. Challenges and strategies for the eradication of the HIV reservoir. Curr Opin Immunol. 2016;42:65-70. 
24. Yukl S, Wong JK. Blood and guts and HIV: preferential HIV persistence in GI mucosa. J Infect Dis. 2008;197(5):640-642.

25. Zhu T, et al. Genetic characterization of human immunodeficiency virus type 1 in blood and genital secretions: evidence for viral compartmentalization and selection during sexual transmission. J Virol. 1996;70(5):3098-3107.

26. Anderson JA, et al. HIV-1 populations in semen arise through multiple mechanisms. PLoS Pathog. 2010;6(8):e1001053.

27. Stekler J, et al. HIV dynamics in seminal plasma during primary HIV infection. AIDS Res Hum Retroviruses. 2008;24(10):1269-1274.

28. Le Tortorec A, Dejucq-Rainsford N. [The male genital tract: A host for HIV]. Gynecol Obstet Fertil. 2007;35(12):1245-1250.

29. Chaillon A, et al. HIV Trafficking Between Blood and Semen During Early Untreated HIV Infection. JAcquir Immune Defic Syndr. 2017;74(1):95-102.

30. Chaillon A, Gianella S, Wertheim JO, Richman DD, Mehta SR, Smith DM. HIV migration between blood and cerebrospinal fluid or semen over time. J Infect Dis. 2014;209(10):1642-1652.

31. Wang TH, Donaldson YK, Brettle RP, Bell JE, Simmonds P. Identification of shared populations of human immunodeficiency virus type 1 infecting microglia and tissue macrophages outside the central nervous system. J Virol. 2001;75(23):11686-11699.

32. Sturdevant CB, Joseph SB, Schnell G, Price RW, Swanstrom R, Spudich S. Compartmentalized replication of R5 T cell-tropic HIV-1 in the central nervous system early in the course of infection. PLoS Pathog. 2015;11(3):e1004720.

33. Strain MC, et al. Genetic composition of human immunodeficiency virus type 1 in cerebrospinal fluid and blood without treatment and during failing antiretroviral therapy. J Virol. 2005;79(3):1772-1788.

34. Stam AJ, Nijhuis M, van den Bergh WM, Wensing AM. Differential genotypic evolution of HIV-1 quasispecies in cerebrospinal fluid and plasma: a systematic review. AIDS Rev. 2013;15(3):152-161.

35. Schnell G, Price RW, Swanstrom R, Spudich S. Compartmentalization and clonal amplification of HIV-1 variants in the cerebrospinal fluid during primary infection. J Virol. 2010;84(5):2395-2407.

36. Oliveira MF, et al. Early antiretroviral therapy is associated with lower HIV DNA molecular diversity and lower inflammation in cerebrospinal fluid but does not prevent the establishment of compartmentalized HIV DNA populations. PLoS Pathog. 2017;13(1):e1006112.

37. Bednar MM, et al. Compartmentalization, viral evolution, and viral latency of HIV in the CNS. Curr HIV/AIDS Rep. 2015;12(2):262-271.

38. Lamers SL, et al. HIV DNA is frequently present within pathologic tissues evaluated at autopsy from combined antiretroviral therapy-treated patients with undetectable viral loads. JVirol. 2016;90(20):8968-8983.

39. Rose R, et al. HIV maintains an evolving and dispersed population in multiple tissues during suppressive combined antiretroviral therapy in individuals with cancer. J Virol. 2016;90(20):8984-8993.

40. De Scheerder MA, et al. HIV rebound Is predomi- nantly fueled by genetically identical viral expansions from diverse reservoirs. Cell Host Microbe. 2019;26(3):347-358.e7.

41. Vásquez JJ, Hunt PW. Participating in human immunodeficiency virus cure research at the end of life. Clin Infect Dis. 2019;69(6):1068-1070.

42. Dubé K, et al. Ethical considerations for HIV cure-related research at the end of life. BMC Med Ethics. 2018;19(1):83.

43. Gianella S, et al. Can research at the end of life be a useful tool to advance HIV cure? AIDS. 2017;31(1):1-4.

44. Bruner KM, et al. Defective proviruses rapidly accumulate during acute HIV-1 infection. Nat Med. 2016;22(9):1043-1049.

45. Pardons M, et al. Single-cell characterization and quantification of translation-competent viral reservoirs in treated and untreated HIV infection. PLoS Pathog. 2019;15(2):e1007619.

46. Tamura K, Nei M. Estimation of the number of nucleotide substitutions in the control region of mitochondrial DNA in humans and chimpanzees. Mol Biol Evol. 1993;10(3):512-526.

47. Chun TW, Moir S, Fauci AS. HIV reservoirs as obstacles and opportunities for an HIV cure. Nat Immunol. 2015;16(6):584-589.

48. van Marle G, Church DL, van der Meer F, Gill MJ. Combating the HIV reservoirs. Biotechnol Genet Eng Rev. 2018;34(1):76-89.

49. Smith MZ, Wightman F, Lewin SR. HIV reservoirs and strategies for eradication. Curr HIV/ AIDS Rep. 2012;9(1):5-15.

50. Yukl SA, et al. The distribution of HIV DNA and RNA in cell subsets differs in gut and blood of HIV-positive patients on ART: implications for viral persistence. J Infect Dis. 2013;208(8):1212-1220.

51. Nolan DJ, et al. The spleen is an HIV-1 sanctuary during combined antiretroviral therapy. AIDS Res Hum Retroviruses. 2018;34(1):123-125.

52. Couturier J, Lewis DE. HIV Persistence in adipose tissue reservoirs. Curr HIV/AIDS Rep. 2018;15(1):60-71

53. Smith DM, Kingery JD, Wong JK, Ignacio CC Richman DD, Little SJ. The prostate as a reservoir for HIV-1. AIDS. 2004;18(11):1600-1602.

54. Anari F, Ramamurthy C, Zibelman M. Impact of tumor microenvironment composition on therapeutic responses and clinical outcomes in cancer. Future Oncol. 2018;14(14):1409-1421.

55. Cohn LB, et al. HIV-1 integration landscape during latent and active infection. Cell. 2015;160(3):420-432.

56. Maldarelli F, et al. HIV latency. Specific HIV integration sites are linked to clonal expansion and persistence of infected cells. Science. 2014;345(6193):179-183.

57. Wagner TA, et al. HIV latency. Proliferation of cells with HIV integrated into cancer genes contributes to persistent infection. Science. 2014;345(6196):570-573

58. Cohn LB, et al. HIV-1 integration landscape during latent and active infection. Cell. 2015;160(3):420-432.

59. Patro SC, et al. Combined HIV-1 sequence and integration site analysis informs viral dynamics and allows reconstruction of replicating viral ancestors. Proc Natl Acad Sci USA 2019;116(51):25891-25899.
60. Telwatte S, et al. Gut and blood differ in constitutive blocks to HIV transcription, suggesting tissue-specific differences in the mechanisms that govern HIV latency. PLoS Pathog. 2018;14(11):e1007357.

61. Yukl S, Wong JK. Blood and guts and HIV: preferential HIV persistence in GI mucosa. J Infect Dis. 2008;197(5):640-642.

62. Embretson J, et al. Massive covert infection of helper $\mathrm{T}$ lymphocytes and macrophages by HIV during the incubation period of AIDS. Nature. 1993;362(6418):359-362.

63. Pantaleo G, et al. HIV infection is active and progressive in lymphoid tissue during the clinically latent stage of disease. Nature. 1993;362(6418):355-358.

64. Wong JK, et al. Reduction of HIV-1 in blood and lymph nodes following potent antiretroviral therapy and the virologic correlates of treatment failure. Proc Natl Acad Sci USA. 1997;94(23):12574-12579.

65. Günthard HF, et al. Residual human immunodeficiency virus (HIV) Type 1 RNA and DNA in lymph nodes and HIV RNA in genital secretions and in cerebrospinal fluid after suppression of viremia for 2 years. J Infect Dis. 2001;183(9):1318-1327.

66. Joos B, et al. HIV rebounds from latently infected cells, rather than from continuing low-level replication. Proc Natl Acad Sci USA 2008;105(43):16725-16730.

67. Gupta RK, et al. HIV-1 remission following CCR5 $\Delta 32 / \Delta 32$ haematopoietic stem-cell transplantation. Nature. 2019;568(7751):244-248

68. Hütter G, et al. Long-term control of HIV by CCR5 delta32/delta32 stem-cell transplantation. N Engl J Med. 2009;360(7):692-698.

69. Barton K, et al. Broad activation of latent HIV-1 in vivo. Nat Commun. 2016;7:12731.

70. Lu CL, et al. Relationship between intact HIV-1 proviruses in circulating $\mathrm{CD}^{+}{ }^{+} \mathrm{T}$ cells and rebound viruses emerging during treatment interruption. Proc Natl Acad Sci USA. 2018;115(48):E11341-E11348.

71. Travers SA, O'Connell MJ, McCormack GP, McInerney JO. Evidence for heterogeneous selective pressures in the evolution of the env gene in different human immunodeficiency virus type 1 subtypes. J Virol. 2005;79(3):1836-1841.

72. Choisy M, Woelk CH, Guégan JF, Robertson DL. Comparative study of adaptive molecular evolution in different human immunodeficiency virus groups and subtypes. JVirol. 2004;78(4):1962-1970.

73. Seibert SA, Howell CY, Hughes MK, Hughes AL. Natural selection on the gag, pol, and env genes of human immunodeficiency virus 1 (HIV-1). Mol Biol Evol. 1995;12(5):803-813.

74. Holmes EC, Zhang LQ, Simmonds P, Ludlam CA, Brown AJ. Convergent and divergent sequence evolution in the surface envelope glycoprotein of human immunodeficiency virus type 1 within a single infected patient. Proc Natl Acad Sci USA. 1992;89(11):4835-4839.

75. Leitner T, Kumar S, Albert J. Tempo and mode of nucleotide substitutions in gag and env gene fragments in human immunodeficiency virus type 1 populations with a known transmission history. J Virol. 1997;71(6):4761-4770.

76. Vrancken B, Alavian SM, Aminy A, Amini-Bavil-Olyaee S, Pourkarim MR. Why com- 
prehensive datasets matter when inferring epidemic links or subgenotyping. Infect Genet Evol. 2018;65:350-351.

77. Schnell G, Price RW, Swanstrom R, Spudich S. Compartmentalization and clonal amplification of HIV-1 variants in the cerebrospinal fluid during primary infection. J Virol. 2010;84(5):2395-2407.

78. Bale MJ, Kearney MF. Review: HIV-1 phylogeny during suppressive antiretroviral therapy. Curr Opin HIV AIDS. 2019;14(3):188-193.

79. Reeves DB, Duke ER, Wagner TA, Palmer SE, Spivak AM, Schiffer JT. A majority of HIV persistence during antiretroviral therapy is due to infected cell proliferation. Nat Commun. 2018;9(1):4811.

80. Pinzone MR, et al. Longitudinal HIV sequencing reveals reservoir expression leading to decay which is obscured by clonal expansion. Nat Commun. 2019;10(1):728.

81. Josefsson L, et al. The HIV-1 reservoir in eight patients on long-term suppressive antiretroviral therapy is stable with few genetic changes over time. Proc Natl Acad Sci USA. 2013;110(51):E4987-E4996.

82. von Stockenstrom S, et al. Longitudinal genetic characterization reveals that cell proliferation maintains a persistent HIV yype 1 DNA pool during effective HIV therapy. JInfect Dis. 2015;212(4):596-607.

83. Wagner TA, McKernan JL, Tobin NH, Tapia KA, Mullins JI, Frenkel LM. An increasing proportion of monotypic HIV-1 DNA sequences during antiretroviral treatment suggests proliferation of HIV-infected cells. J Virol. 2013;87(3):1770-1778.

84. Hosmane NN, et al. Proliferation of latently infected $\mathrm{CD} 4^{+} \mathrm{T}$ cells carrying replication-competent HIV-1: potential role in latent reservoir dynamics. J Exp Med. 2017;214(4):959-972.

85. Laskey SB, Pohlmeyer CW, Bruner KM, Siliciano RF. Evaluating clonal expansion of HIV-infected cells: optimization of PCR strategies to predict clonality. PLoS Pathog. 2016;12(8):e1005689.

86 . Hiener B, et al. Identification of genetically Intact $\mathrm{HIV}-1$ proviruses in specific $\mathrm{CD} 4^{+} \mathrm{T}$ cells from effectively treated participants. Cell Rep. 2017;21(3):813-822.

87. Bruner KM, et al. Defective proviruses rapidly accumulate during acute HIV-1 infection. Nat Med. 2016;22(9):1043-1049.

88. Lee GQ, et al. Clonal expansion of genome-intact HIV-1 in functionally polarized Th1 CD4+ T cells. JClin Invest. 2017;127(7):2689-2696.
89. Weinberger AD, Perelson AS. Persistence and emergence of X4 virus in HIV infection. Math Biosci Eng. 2011;8(2):605-626.

90. Allers K, et al. Evidence for the cure of HIV infection by CCR $5 \Delta 32 / \Delta 32$ stem cell transplantation. Blood. 2011;117(10):2791-2799.

91. Julg B, et al. Recommendations for analytical antiretroviral treatment interruptions in HIV research trials-report of a consensus meeting. Lancet HIV. 2019;6(4):e259-e268.

92. Quinn GP, et al. Altruism in terminal cancer patients and rapid tissue donation program: does the theory apply? Med Health Care Philos. 2013;16(4):857-864.

93. Lintz KC, Penson RT, Chabner BA, Mack S, Lynch TJ. Schwartz Center rounds. a staff dialogue on phase I trials: psychosocial issues faced by patients, their families, and caregivers. Oncologist. 1998;3(5):357-364.

94. Strain MC, et al. Highly precise measurement of HIV DNA by droplet digital PCR. PLoS One. 2013;8(4):e55943.

95. Pinheiro LB, et al. Evaluation of a droplet digital polymerase chain reaction format for DNA copy number quantification. Anal Chem. 2012;84(2):1003-1011.

96. Ho YC, et al. Replication-competent noninduced proviruses in the latent reservoir increase barrier to HIV-1 cure. Cell. 2013;155(3):540-551.

97. Nguyen LT, Schmidt HA, von Haeseler A, Minh BQ. IQ-TREE: a fast and effective stochastic algorithm for estimating maximum-likelihood phylogenies. Mol Biol Evol. 2015;32(1):268-274.

98. Pinzone MR, et al. Longitudinal HIV sequencing reveals reservoir expression leading to decay which is obscured by clonal expansion. Nat Commun. 2019;10(1):728.

99. Rose PP, Korber BT. Detecting hypermutations in viral sequences with an emphasis on $\mathrm{G} \rightarrow$ A hypermutation. Bioinformatics. 2000;16(4):400-401.

100.[No authors listed]. Los Alamos National Laboratory, and National Institutes of Health. HIV databases. http://www.hiv.lanl.gov/. Accessed January 27, 2020.

101.HIV Los Alamos National Laboratory. ElimDupes. https://www.hiv.lanl.gov/content/ sequence/elimdupesv2/elimdupes.html.

102. Beerenwinkel N, et al. Geno2pheno: estimating phenotypic drug resistance from HIV-1 genotypes. Nucleic Acids Res. 2003;31(13):3850-3855.
103. Parker J, Rambaut A, Pybus OG. Correlating viral phenotypes with phylogeny: accounting for phylogenetic uncertainty. Infect Genet Evol. 2008;8(3):239-246.

104.Suchard MA, Lemey P, Baele G, Ayres DL, Drummond AJ, Rambaut A. Bayesian phylogenetic and phylodynamic data integration using BEAST 1.10. Virus Evol. 2018;4(1):vey016.

105. Minin VN, Bloomquist EW, Suchard MA. Smooth skyride through a rough skyline: Bayesian coalescent-based inference of population dynamics. Mol Biol Evol. 2008;25(7):1459-1471.

106.Drummond AJ, Ho SY, Phillips MJ, Rambaut A. Relaxed phylogenetics and dating with confidence. PLoS Biol. 2006;4(5):e88.

107. Lemey P, Rambaut A, Drummond AJ, Suchard MA. Bayesian phylogeography finds its roots. PLoS Comput Biol. 2009;5(9):e1000520.

108. Edwards CJ, et al. Ancient hybridization and an Irish origin for the modern polar bear matriline. Curr Biol. 2011;21(15):1251-1258.

109. Bielejec F, Baele G, Vrancken B, Suchard MA, Rambaut A, Lemey P. SpreaD3: interactive visualization of spatiotemporal history and trait evolutionary processes. Mol Biol Evol. 2016;33(8):2167-2169.

110. Kass RE, Raftery AE. Bayes Factors. J Am Stat Assoc. 1995;90(430):773-795.

111. Minin VN, Suchard MA. Counting labeled transitions in continuous-time Markov models of evolution. JMath Biol. 2008;56(3):391-412.

112. Faria NR, et al. HIV epidemiology. The early spread and epidemic ignition of HIV-1 in human populations. Science. 2014;346(6205):56-61.

113. Firth C, Kitchen A, Shapiro B, Suchard MA, Holmes EC, Rambaut A. Using time-structured data to estimate evolutionary rates of double-stranded DNA viruses. Mol Biol Evol. 2010;27(9):2038-2051.

114. Trovão NS, et al. Host ecology determines the dispersal patterns of a plant virus. Virus Evol. 2015;1(1):vev016.

115. Rambaut A, Drummond AJ, Xie D, Baele G, Suchard MA. Posterior summarization in Bayesian phylogenetics using Tracer 1.7. Syst Biol. 2018;67(5):901-904.

116. Lemey P, et al. Unifying viral genetics and human transportation data to predict the global transmission dynamics of human influenza H3N2. PLoS Pathog. 2014;10(2):e1003932.

117. Danielson MM, Dubé K. Michael's testimonial. Ann Intern Med. 2019;170(7):511-512. 